two patient groups also limits interpretation. The recruitment of consecutive patients, with its inherent variability, can be seen as a strength of the study, but it also creates problems due to the range of disease seen-for example, patients' mini mental state examination scores ranged from 4 to 26 . It is therefore not clear how confident one can be about the positive findings irrespective of disease duration or severity given the few patients at each data point. Indeed it could be argued that comparing only mildly affected patients may be more useful as it is this group that represent the greatest challenge both diagnostically and therapeutically - although the absence of effective long term therapies may even limit this potential at the present time.

In conclusion, this study highlights the possibility that neurodegenerative disorders targeting the cerebral cortex may be distinguished through their effects on sympathetic nerve endings in the heart. However, perhaps the most important message from this study is to highlight once more that neurodegenerative disorders display widespread pathology and that this needs to be accommodated in any theory of pathogenesis as well as novel therapeutic approaches.

R A BARKER

Department of Neurology and Cambridge Centre for Brain Repair, Addenbrooke's Hospital, Hills Road, Cambridge CB2 2QQ, UK

rab46@cus.cam.ac.uk

1 Yoshita M, Taki J, Yamada M. A clinical role for ${ }^{123}$ I MIBG myocardial scintigraphy in the distinction between dementia of the Alzheimer's type and dementia with lewy bodies. J Neurol Neurosurg Psychiatry 2001;71:583-8.

2 Yoshita M. Differentiation of idiopathic Parkinson's disease from striatonigral degeneration and progressive supranuclear palsy using iodine-123metaiodobenzylguanidine myocardial scintigraphy. J Neurol Sci 1998;155: 60-7.

3 Druschky A, Hilz MJ, Platsch G, et al. Differentiation of Parkinson's disease and multiple system atrophy in early disease stages by means of I-123-MIBG-SPECT. J Neurol Sci 2000;175:3-12

4 Stoddard SL, Ahlskog JE, Kelly PJ et al. Decreased adrenal medullary catecholamines in adrenal transplanted parkinsonian patients compared to nephrectomy patients. Exp Neurol 1989;104:218-22.

\title{
Videodynamic and sphincter motor unit potential analyses in Parkinson's disease and multiple system atrophy
}

In the paper by Sakakibara et al (this volune, pp 600-606), ${ }^{1}$ the differences in findings of investigations of bladder function between patients with Parkinson's disease and multiple system atrophy are reported. The authors' new findings focus on the urethral sphincter mechanism, examining this functionally (using some of the newer urodynamic indices) and fluoroscopically. But the paper also provides a useful summary, reviewing as it does, previous work in this area, which has so far been mainly published in the urological literature. Although urinary symptoms can be troublesome in advanced Parkinson's disease they do not have the same prominence and severity as those seen in early multiple system atrophy, probably due to the multiple defects of neurological control of the bladder and sphincter function that develop in the initial stages of multiple system atrophy. Abnormalities of urethral sphincter innervation, both the external (striated) and intrinsic (bladder neck) are marked as a feature of multiple system atrophy but not Parkinson's disease, as has been shown in this study.

There has been a tendency up to now to include bladder symptoms as part of the "autonomic failure" which characterises multiple system atrophy, but the growing realisation that the entire system of neurological control of the bladder is selectively involved in its early stages, may lead to a new approach in understanding the evolution of this progressive and fatal neurodegenerative disease.

C J FOWLER

Department of Uro-Neurology, National Hospital for Neurology and Neurosurgery, Queen Square, London WC1N 3BG, UK

c.fowler@ion.ucl.ac.uk

1 Sakakibara R, Hattori T, Uchiyama T, et al. Videodynamic and sphincter motor unit potential analyses in Parkinson's disease and multiple system atrophy. $\mathcal{F}$ Neurol Neursurg Psychiatry 2001;71:600-6.

\section{Sphincter EMG in possible multiple system atrophy: to do or not to do?}

In multiple system atrophy (MSA) the motor neurons of sphincter muscles (Onuf's nucleus) degenerate earlier than those of limb muscles (Sung et $a l^{1}$ ). Such lower motor neuron loss is, in principle, accompanied by muscle pathology, which may be readily assessed by EMG methods, of which there are several. Although there is no doubt that expertise may be accumulated with any of the various methods, data comparing different techniques are only beginning to appear. Gilad et al (this issue, pp 596-99) report on results obtained by concentric needle EMG (CNEMG) single fibre EMG (SFEMG), and interference pattern (IP) analysis in a small group of patients with MSA. ${ }^{2}$ Their findings both confirm and contradict the commonly held views on sphincter EMG findings in MSA. As expected, some EMG abnormalities were found in all patients studied, but the reported changes in isolation (reduced 
recruitment pattern with a reduced number of active motor units during rest) are not specific for loss of lower motor neurons. Comparing patients with MSA and healthy controls, no significant differences in CNEMG and SFEMG parameters (which are known to correlate with reinnervation changes in muscle) were found. Thus, the "new" parameter may either be more sensitive in detecting early degeneration of Onuf's nucleus, or reflect upper motor neuron involvement. The failure of Gilad et $a t^{2}$ to detect significant "classic" EMG abnormalities cannot be due to insensitivity of their CNEMG analysis (although the "multi-motor unit potential (multi-MUP) technique" has problems with detection of unstable complex MUPs, held to be typical of MSA) because of their confirmatory SFEMG findings. Thus, their results can actually be interpreted as showing that many patients with "possible" or "probable" MSA have no degeneration of Onuf's nucleus! However, practically all previous studies have demonstrated obvious ("classic") EMG abnormalities! The dissenting voices in the literature do not question the presence of EMG abnormalities in MSA, but whether these are significantly different from those in other degenerative extrapyramidal diseases (the literature is ample and has recently been reviewed- see Vodušek ${ }^{3}$ ). The results of Gilad et $a l^{2}$ thus question the value of "classic" sphincter EMG more radically (however, based on results of only 11 patients). The "majority opinion" of studies of a total of well over 500 patients with MSA still supports the "expert opinion" that the finding of pathological spontaneous activity and/or significant MUP abnormalities in the sphincter muscle favours - in the appropriate clinical setting - the diagnosis of MSA. The "appropriate setting" includes caveats that the patient with parkinsonism should be in the first 5 years of the disease course, and that he or she has no other cause for sphincter denervation; EMG does not differentiate between MSA and progressive supranuclear palsy. The absence of EMG abnormalities, however - and this was appreciated even before the article by Gilad et $a l^{2}$ - does not rule out MSA (see Vodušek ${ }^{3}$ ).

The techniques of EMG and the assessed parameters used up until now have varied, and thoroughly explored normative data from a large control group with a well standardised method of quantitative CNEMG have only recently been published-by Podnar et $a l,{ }^{4}$ and now by Gilad et al. The method ("multi-MUP") allows standardised quantitative assessment of a sizeable sample of motor unit potentials (MUPs) with little personal bias. With IP analysis there is even less personal bias, with Gilad et al stressing the informative value of this method in patients with MSA. It seems that the above mentioned methods should be the logical choice in future studies, which should clarify the time course of sphincter motor neuron degeneration in MSA, preferably combining methods demonstrating functional and structural changes.

D B VODUŠEK

Division of Neurology, University Medical Centre, Zaloška 7, 1525

Ljubljana, Slovenia

david.vodusek.@kcl.si

1 Sung J, Mastri A, Segal E. Pathology of Shy-Drager syndrome. J Neuropathol Exp Neurol 1979;49:353-68.

2 Gilad R, Giladi N, Korczyn AD, et al. Quantitative anal sphincter EMG in multisystem atrophy and one hundred controls. J Neurol Neurosurg Psychiatry 2001;71:596-9.

3 Vodušek DB. Sphincter EMG and differential diagnosis of multiple system atrophy. Mov Dis $2001 ; 16: 600-7$.

4 Podnar S, Vodušek DB, Stålberg E. Standardization of anal sphincter electromyography: normative data. Clin Neurophysiol 2000;111:2200-7. 